\title{
Correction to: Commensurability classes of fake quadrics
}

\author{
Benjamin Linowitz ${ }^{1} \cdot$ Matthew Stover ${ }^{2} \cdot$ John Voight ${ }^{3}$
}

Published online: 6 September 2019

๑) Springer Nature Switzerland AG 2019

\section{Correction to: Selecta Mathematica (2019) 25:48 https://doi.org/10.1007/s00029-019-0492-9}

In the original publication of the article, the author comment was misunderstood and the section headings were updated incorrectly. Now, the section headings have been corrected in the original article as given below:

1) Introduction

2) Background

2.1) Geometry of fake quadrics

2.2) Number fields and quaternion algebras

3) Arithmetic lattices and quaternion algebras

3.1) Lattices potentially yielding fake quadrics

3.2) Stable subgroups

3.3) Maximal arithmetic lattices and torsion

4) Generalizing a volume inequality of Chinburg and Friedman

5) Enumerating fields and fake quadrics

5.1) Further restrictions on $k$

The original article can be found online at https://doi.org/10.1007/s00029-019-0492-9.

$凶 \quad$ Matthew Stover

mstover@temple.edu

Benjamin Linowitz

benjamin.linowitz@oberlin.edu

John Voight

jvoight@gmail.com

1 Department of Mathematics, Oberlin College, 10 North Professor Street, Oberlin, OH 44074, USA

2 Department of Mathematics, Temple University, 1805 N. Broad Street, Philadelphia, PA 19122, USA

3 Department of Mathematics, Dartmouth College, 6188 Kemeny Hall, Hanover, NH 03755, USA 


\section{2) Enumerating maximal arithmetic subgroups containing fake quadrics}

Publisher's Note Springer Nature remains neutral with regard to jurisdictional claims in published maps and institutional affiliations. 